\title{
Viewing Angle of Binary Neutron Star Mergers
}

\author{
Hsin-Yu Chen, ${ }^{1, *}$ Salvatore Vitale, ${ }^{2, \dagger}$ and Ramesh Narayan ${ }^{3, *}$ \\ ${ }^{1}$ Black Hole Initiative, Harvard University, Cambridge, Massachusetts 02138, USA \\ ${ }^{2}$ LIGO Laboratory and Kavli Institute for Astrophysics and Space Research, \\ Massachusetts Institute of Technology, Cambridge, Massachusetts 02139, USA \\ ${ }^{3}$ Harvard-Smithsonian Center for Astrophysics, Harvard University, \\ Cambridge, Massachusetts 02138, USA
}

(Received 26 July 2018; revised manuscript received 14 July 2019; published 19 August 2019)

\begin{abstract}
The joint detection of the gravitational wave (GW) GW170817 and its electromagnetic (EM) counterparts GRB170817A and kilonova AT 2017gfo has triggered extensive study of the EM emission of binary neutron star mergers. A parameter which is common to and plays a key role in both the GW and the EM analyses is the viewing angle of the binary's orbit. If a binary is viewed from different angles, the amount of GW energy changes (implying that orientation and distance are correlated) and the EM signatures can vary, depending on the structure of the emission. Information about the viewing angle of the binary orbital plane is therefore crucial to the interpretation of both the GW and the EM data and can potentially be extracted from either side. In the first part of this study, we present a systematic analysis of how well the viewing angle of binary neutron stars can be measured from the GW data. We show that if the sky position and the redshift of the binary can be identified via the EM counterpart and an associated host galaxy, then for $50 \%$ of the systems the viewing angle can be constrained to $\leq 7^{\circ}$ uncertainty from the GW data, independent of electromagnetic emission models. On the other hand, if no redshift measurement is available, the measurement of the viewing angle with GWs alone is not informative, unless the true viewing angle is close to $90^{\circ}$. This holds true even if the sky position is measured independently. Then, we consider the case where some constraints on the viewing angle can be placed from the EM data themselves. We show that the EM measurements can then be used in the analysis of GW data to improve the precision of the luminosity distance, and hence of the Hubble constant, by a factor of 2-3.
\end{abstract}

DOI: 10.1103/PhysRevX.9.031028

\section{INTRODUCTION}

The discovery of binary neutron star (BNS) merger GW170817 [1] and its electromagnetic (EM) counterparts, GRB170817A and kilonova AT 2017gfo, opened the era of multimessenger astronomy [2-5].

While much was learned from this first joint detection, the precise EM emission model is still unknown. A key parameter to understand the EM emission is the orbital inclination angle, [6] which strongly impacts the details of the EM signals received at Earth. For example, the gammaray burst (GRB) GRB170817A is underluminous [3,7], and the $\mathrm{x}$-ray and radio emission that followed are significantly different from those of other GRBs [8-12]. A possible

\footnotetext{
*hsinyuchen@fas.harvard.edu

†salvatore.vitale@ligo.mit.edu

*narayan@cfa.harvard.edu
}

Published by the American Physical Society under the terms of the Creative Commons Attribution 4.0 International license. Further distribution of this work must maintain attribution to the author(s) and the published article's title, journal citation, and DOI.
Subject Areas: Astrophysics, Cosmology, Gravitation

explanation could be that a GRB jet was formed, which we observe from a large inclination angle. However, alternative explanations exist. The observations could also be described by a choked jet, whose cocoon expands, leading to a wide-angle, mildly relativistic outflow $[13,14]$. If the choked jet is the correct scenario, the inclination angle affects less the light curve and the spectra and, thus, cannot be constrained by the available EM data. Although recent follow-up observations seem to favor the off-axis emission explanation for GW170817/GRB170817A [15-17], other models have not definitively been ruled out and will be tested with future joint detections. The inclination angle is also a key ingredient for the interpretation of the kilonova emission and its spectral evolution [9,18-21].

It would thus be important if the inclination angle of the binary, $\theta_{\mathrm{JN}}$, could be measured or at least constrained from the GW data and then used to study or exclude EM emission mechanisms.

Unfortunately, measurement of the inclination angle with GW data is usually quite poor, due to the well-known degeneracy between the inclination and the luminosity distance [22]. This degeneracy can be resolved if the system has precessing spins, if higher-order harmonics 
are detectable, or if the merger and ringdown are in band. None of these conditions are met for BNSs, since neutron stars have small spins and mass ratios close to one, which suppresses the amplitude of higher-order harmonics [23-29]. However, in the case of a joint GW-EM detection, more information usually exists. If an independent measurement of the luminosity distance can be made from the redshift of the EM counterpart or using other properties of the host [30], the degeneracy is broken, and one can expect the uncertainty on the inclination angle to improve. To a smaller extent, knowledge of sky position can also help to improve the measurement of the inclination angle, as we discuss below. One could thus envisage a strategy where some information obtained from the EM analysis (redshift, sky position) can be folded into the GW analysis to get an improved measurement of the inclination angle. We note that, although $\mathrm{GW}$ detectors are able to measure the binary inclination angle $\theta_{\mathrm{JN}}$, which also carries directional information on the binary rotation (clockwise or counterclockwise) and has a range between $0^{\circ}$ and $180^{\circ}$, most EM observations depend on only the binary viewing angle $\zeta$, which is defined as $\zeta \equiv \min \left(\theta_{\mathrm{JN}}, 180^{\circ}-\theta_{\mathrm{JN}}\right)$ [3]. We hereby focus on the viewing angle in this paper. Using the sky position of the kilonova AT 2017gfo and the redshift of the host galaxy NGC4993, the bound on the viewing angle of GW170817 is improved from $\zeta \lesssim 56^{\circ}[1]$ to $\zeta \lesssim 28^{\circ}[1,31,32]$.

In the future, one might also consider the opposite approach: If the details of the EM emission are well understood, the detection of photons could provide a bound on the viewing angle. For example, detection of a short GRB with a jet break in the afterglow can yield an upper bound on the binary viewing angle. The jet break indicates that we observe the short GRB within the core of its relativistic jet [33-36]. If the jet is aligned with the binary rotation axis, the jet break constrains the maximum viewing angle of the binary. In addition, the observations of GRB170817A and the kilonova AT 2017gfo have led to more thorough studies on the EM emission models, providing measurements of GW170817's viewing angle even without the detection of a jet break (e.g., Refs. [10,15,37]). This information can be used in the GW analysis to get a better measurement of the luminosity distance [38], which, in turn, can improve the Hubble constant measurement with GWs $[39,40]$.

In this paper, we present a systematic study of the measurability of the viewing angle of BNS systems. We first show that, in the absence of a positive detection of an EM counterpart, GWs alone only rarely provide a meaningful constraint on the viewing angle. We then consider the case when the luminosity distance and/or the sky position are independently measured. While little is changed by knowing the sky position, knowledge of the luminosity distance dramatically reduces the $1 \sigma$ uncertainty for the viewing angle. For 50\% of the BNS detections for which the sky position and luminosity distance are measured from EM data, the viewing angle uncertainty is below $7^{\circ}$ (using the projected sensitivities for LIGO and Virgo in the third science run). This uncertainty is small enough to allow for an interesting comparison to EM emission models.

In this paper, we present a systematic study of the measurement of the GW binary viewing angle using auxiliary EM information. The end-to-end set of simulations we perform was not previously possible due to the expensive computational costs required to carry fully numerical Markov chain Monte Carlo (MCMC) parameter estimation runs for a large number of sources [41]. We circumvent this issue by running the full MCMC algorithm [41] for a subset of sources and show how a different and faster dedicated code we have written yields the same results. This faster code is what allows us to carry out the study we describe in this paper.

We also consider the opposite scenario and show that, if the binary viewing angle is constrained by the EM data, the binary luminosity distance uncertainty can potentially be reduced by a factor of $2-3$. In turn, that improves the measurement of the Hubble constant with GWs. We show that a $1 \%$ uncertainty on the Hubble constant can then be reached with only $O(10)$ GW-EM joint detections of BNSs, that is, in less than $5 \mathrm{yr}$.

We note that Refs. $[38,42,43]$ discuss the improvement on the Hubble constant measurement with GWs when a short GRB counterpart is found. In these studies, the jet is assumed to be aligned with the binary rotation axis, and the short GRB observations provide upper bounds for the binary viewing angle (in the form of a top-hat prior or some other probability distribution, usually with a maximum at $\zeta=0^{\circ}$, and decreasing at higher angles). In one of the analyses we carry out, we consider different values for the upper bound on the viewing angle from EM observations. However, we find that the resulting improvement in the distance measurement does not strongly depend on how tight the upper bound is, as long as the bound is below approximately $30^{\circ}$. In addition to this upper-bound approach, we also consider a new and more realistic scenario where the binary viewing angle is not constrained from $0^{\circ}$ to some upper bound but rather is given as a normal distribution centered at different values (from $0^{\circ}$ to $90^{\circ}$ ). This extends what was done in previous literature, which exclusively focuses on small viewing angles. We argue that it is important to not only focus on a small viewing angle, since future events will usually have moderate viewing angles (approximately $30^{\circ}$ ) [44]. Even with a viewing angle as large as $30^{\circ}$, EM observations can still yield meaningful constraints, as shown by the follow-up of GW170817.

\section{METHOD AND RESULTS}

We consider two different methods to estimate the distance and inclination of BNS systems. First, we rely on the computationally expensive stochastic sampler 
LALINFERENCE [41] (specifically on its nested sampling flavor [45]). This is the same algorithm used by the LIGO and Virgo Collaborations and delivers posterior distributions for all the unknown parameters on which compact binaries depend. Given the cost of each simulation [41], LALINFERENCE cannot be run on an arbitrarily large number of simulations. We thus use it only on a few specific sources, to show which parameters affect the measurability of luminosity distance and inclination and how. We then introduce a semianalytical, faster, approach. After showing that the two give consistent results, we use the latter to characterize the population of detectable BNSs.

\section{A. Single-event analysis}

All the BNS systems we simulate have component masses 1.4-1.4 $\mathrm{M}_{\odot}$ in the source frame. As mass measured in the detector frame is redshifted by a factor of $(1+z)$, where $z$ is the redshift, the sources will appear slightly heavier in the detector frame.

To keep the computational cost reasonable, we make two main simplifying assumptions: We neglect tidal effects and neutron star spins. The former is a very reasonable choice, since tidal effects do not enter the waveform amplitude and, hence, are not correlated with the inclination angle. The latter is justified, since the spins of known neutron stars in binaries that will merge within a Hubble time is small (the fastest-spinning systems are PSR J0737-3039A [46] and PSR J1946 + 2052 [47], which will at most have dimensionless spins of $\chi \sim 0.04$ or $\chi \sim 0.05$ when they merge). Even GW170817 is consistent with having small spins [31].

In the work reported here, all the synthetic BNS signals are generated using the IMRPHENOMPV waveform family $[48,49]$, with the reduced order quadrature likelihood approximation [50]. We consider a network consisting of the two LIGOs [51] and Virgo [52], all at design sensitivity. The signals are added into "zero noise" (which yields the same results that would be obtained averaging over many noise realizations). We start all analyses at $20 \mathrm{~Hz}$ and use a sampling rate of $8 \mathrm{KHz}$. We marginalize over calibration errors using the same method described in Ref. [53], using Gaussian priors with widths of $3 \%$ for the amplitude and $1.5^{\circ}$ phase for all instruments.

We consider two different sky positions, to verify if and to what extent our conclusions depend on the detector antenna patterns. In total, we create four such systems. Their parameters are summarized in Table I. For sources

TABLE I. For all events the GPS time is 1068936994.0 , and the two component masses in the source frame are $1.4 \mathrm{M}_{\odot}$.

\begin{tabular}{lcclcl}
\hline \hline & SNR & Polarization & Phase & RA & DEC \\
\hline Source $A$ & 35 & 0.005 & 0 & -1.08 & 0.66 \\
Source $B$ & 20 & 0.005 & 0 & -1.08 & 0.66 \\
Source $C$ & 20 & 0.005 & 0 & 0 & $\pi / 2$ \\
Source $D$ & 12 & 0.017 & 0.017 & -1.08 & 0.66 \\
\hline \hline
\end{tabular}

$A, B$, and $D$, the sky position is near the maximum of LIGO's antenna pattern, where one would expect most detections to be made [54].

The main goal of the present study is to verify how well the inclination angle can be measured. Obviously, this would a priori depend on three main factors: the SNR of the event, the true value of the inclination angle, and the sky location of the event. Each of the sources listed in Table I is reanalyzed for different values of the inclination angle, from nearly face on $\left(\theta_{\mathrm{JN}}=0^{\circ}\right)$ to edge on $\left(\theta_{\mathrm{JN}}=90^{\circ}\right)$. Every time the orientation angle is changed, the distance is also varied to keep the SNR fixed at the value given in Table I.

We note that one expects most detections made by advanced detectors to have inclinations close to $30^{\circ}$ or $150^{\circ}$, whereas events with an inclination close to edge on $\left(\theta_{\mathrm{JN}}=90^{\circ}\right)$ would be rarer [44]. Why this happens is related to the degeneracy between the luminosity distance and inclination. Since that will play a role in the interpretation of the results we present, it is worth expanding on the subject. Geometrical arguments would suggest that the inclination angles of the population of binaries should be uniform on the sphere, i.e., $p\left(\theta_{\mathrm{JN}}\right) \sim \sin \left(\theta_{\mathrm{JN}}\right)$, while their luminosity distances should be uniform in volume, $p\left(D_{L}\right) \sim$ $D_{L}^{2}$ (as long as cosmological effects can be neglected). However, the detected binaries are a subset of the entire population: They are the fraction that produce a GW signal loud enough to be detected. GW emission from a compact binary is not isotropic; instead, more energy is emitted along the direction of the orbital angular momentum, while the least amount is emitted parallel to the orbital plane [55]. This implies that edge-on systems, which are the most numerous in the underlying population, will need to be extremely close to be detectable. Conversely, face-on and -off systems can be farther away and still produce a detectable signal. But since far away there is more volume, the population of detectable signals will be dominated by sources with inclination angles close to $30^{\circ}$ and $150^{\circ}$. Note that all events LIGO and Virgo have detected thus far (including the binary black holes) are consistent with $30^{\circ}$ or $150^{\circ}$ inclination angles [22,31,56-60]. The analytical form of the inclination angle distribution for sources detectable by advanced detectors [61] was first obtained by Ref. [44], and we refer to it as the Schutz distribution in this work. Using the Schutz distribution, one can calculate the fraction of detectable events that will have a viewing angle within a given range. In particular, less than approximately $7 \%$ (approximately $3 \%$ ) of the detectable events have a viewing angle $>70^{\circ}\left(>80^{\circ}\right)$.

All analyses are performed three times: a first time assuming that all parameters are unknown and measured from GW data alone; a second time assuming a counterpart is found, which provides the sky position (right ascension and declination) of the source; a third time, assuming both sky position and distance are known (the latter by 
measuring the redshift and using a cosmology to convert redshift to luminosity distance).

For all parameters, we use the same priors used by the LIGO and Virgo Collaborations. In particular, the prior on the luminosity distance is uniform in volume, while the prior on the inclination angle is isotropic.

Since the EM emission depends only on the viewing angle, we quote results for the viewing angle $\zeta$, rather than the inclination angle $\theta_{\mathrm{JN}}$ itself. In Fig. 1 , we report the $1 \sigma$ uncertainty (in degrees) for the measurement of the viewing angle $\zeta$ for all sources, as a function of the true inclination angle.

We first discuss the case where no information is available from the EM side (dot-dashed lines in Fig. 1). We find that the uncertainties are roughly constant until the true inclination angle gets above approximately $80^{\circ}$. For the sources $A, B$, and $C$, the uncertainty happens to be nearly the same: approximately $15^{\circ}$, while it is a little wider for source $D \sim 19.5^{\circ}$. We verify that for all these configurations the viewing angle posterior is not very informative. It is similar to the Schutz distribution, with some extra support at $30^{\circ}$ and a depletion of support close to edge on. This situation is shown in Fig. 2 for source $B$, and in Figs. 8-10 in the Appendix for the other sources.

The fact that the viewing angle uncertainty is the same for small to moderate inclinations can be explained by a combination of priors and the well-known degeneracy between the luminosity distance and inclination. Since the emission of GWs is larger toward the direction of the system angular momentum, one can obtain a similar signal at the detector by increasing the viewing angle while moving the source closer. As the true inclination increases from zero, the true distance has to decrease to maintain the same SNR. However, smaller distances are disfavored by the prior. Thus, the Bayesian code prefers to keep the viewing angle posterior close to face on and overestimate the distance to compensate, which can be done because of the correlation between the two parameters: The eventual

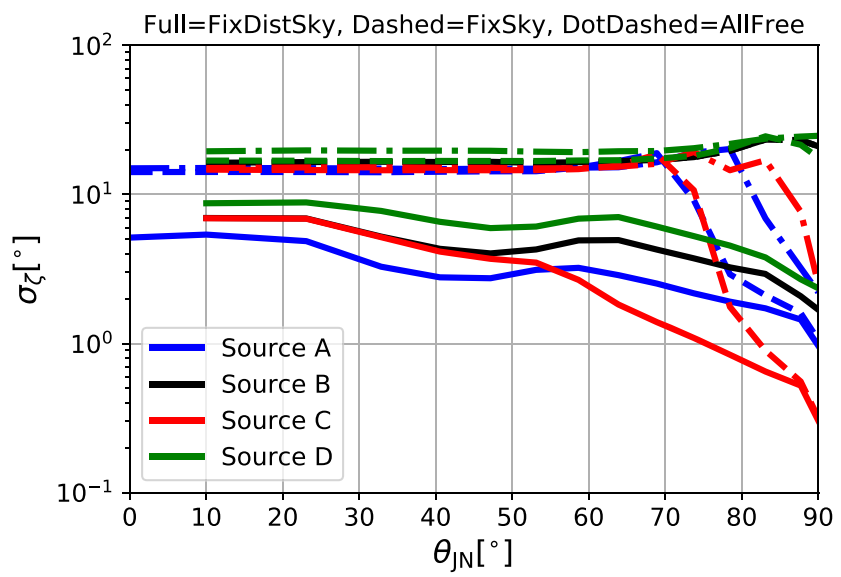

FIG. 1. Viewing angle uncertainty as a function of the binary inclination angle.

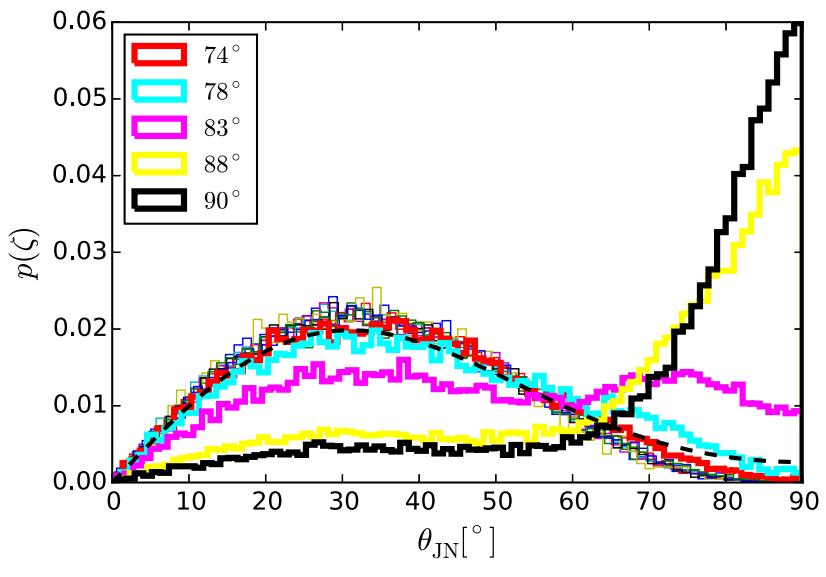

FIG. 2. Viewing angle posteriors for source $B$, assuming no information is provided from the EM sector. The dashed line is obtained from the Schutz distribution. For all values of the inclination angle below approximately $75^{\circ}$, the posteriors are the same (thin lines) and similar to the Schutz distribution. We highlight posteriors for sources with a large viewing angle with thicker lines, where the value of the viewing angle is given in the legend.

small decrease in the likelihood introduced by biasing both the luminosity distance and inclination is more than compensated for by the better prior value at larger distances. This behavior can be sustained till the true inclination angle is close to edge on. At that point, the degeneracy is reduced, and the likelihood penalty for keeping the posterior at face on cannot be compensated for by the prior: Both the luminosity distance and viewing angle posteriors are centered at the true value and typically better measured [63].

For the low SNR event, source $D$, one gets exactly the Schutz distribution for small to moderate inclinations. Unlike for the other sources, in this case the uncertainty increases as the inclination angle gets close to $90^{\circ}$. This increase happens because when the true inclination is close to edge on a significant posterior peak still survives at $30^{\circ}$ : Since the SNR is low, the extra likelihood to be gained with more support at edge on is comparable with the prior penalty, and a bimodal distribution arises (see Fig. 10 in the Appendix).

We find that similar conclusions can be drawn if the sky position of the source can be considered as known (dashed lines in Fig. 1). It is still the case that the posteriors for small to moderate inclinations are similar to the Schutz distribution, and the main difference is that the distanceinclination degeneracy is resolved at smaller inclinations, $\theta_{\mathrm{JN}} \lesssim 70^{\circ}$ (instead of $\theta_{\mathrm{JN}} \lesssim 80^{\circ}$ for the previous case).

It is only when both the sky position and distance are known (solid lines in Fig. 1) that the uncertainties are much smaller for all sources and all inclinations. In this case, the posteriors for the viewing angle are centered around the true value for all systems. The uncertainties reach a minimum when the true source is edge on, since in that case the cross polarization of the GW signals is zero, which 
reduces the residual degeneracy between the inclination and polarization angle $\psi$ (the only two unknown angles left in the amplitude of the GW signal).

It is interesting to verify how precisely the BNS sources can be localized as a function of their orientation. The localization is shown in Fig. 3 for the runs in which all parameters are considered unknown. We find that the $90 \%$ credible interval (in $\mathrm{deg}^{2}$ ) is roughly constant for small to moderate inclination angles and for all sources. Source $A$ is localized better than source $D$ because of its higher SNR. The difference between sources $B$ and $C$ (which have the same network SNR) is that source $C$ has an SNR roughly split in equal amounts in the three interferometers, while source $B$ has most of the SNR in the two LIGOs, while being subthreshold SNR in Virgo (4.2 for the face-on orientation). Since most of the sky resolution for GW sources comes from triangulation and by requiring phase and amplitude consistency across the network [64-66], it helps if the source is above the threshold in more detectors (incidentally, we stress that the same is not true for the viewing angle uncertainty: as Fig. 1 shows, one gets the same uncertainties for sources $B$ and $C$. Likewise, the uncertainty for the luminosity distance is the same for the two sources up to viewing angles of approximately $70^{\circ}$ ). We note that the edge-on sources are relatively poorly localized. This result can be explained as follows: The sky position (right ascension and declination) and orientation (inclination, polarization) angles all enter the frequencydomain GW amplitude and phase [e.g., Eqs. (4.1)-(4.5) in Ref. [67] ]. When the inclination angle is close to $90^{\circ}$, some of these terms are suppressed, reducing the number of constraints that can be used to enforce phase and amplitude consistency, leading to larger uncertainties.

\section{B. Computation considerations for a population analysis}

Having gained an understanding of which parameters can impact the measurability of the viewing angle, we

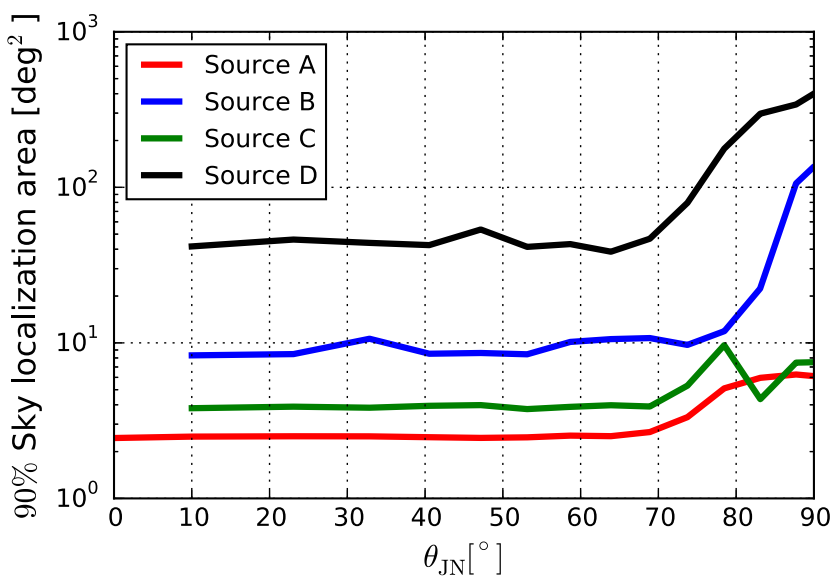

FIG. 3. Sky localization area (in $\mathrm{deg}^{2}$ ) as measured by LALINFERENCE, as a function of the binary inclination angle. determine what fraction of the BNSs for which an EM counterpart is found yield significant viewing angle constraints. Unfortunately, it is computationally prohibitive to run LALINFERENCE on large sets of hundreds or thousands of events.

We thus build an approximate Bayesian estimator for the two binary parameters of interest: the inclination angle and the luminosity distance.

The algorithm assumes that the sky position, chirp mass, and mass ratio of the binaries are known. These assumptions can be justified as follows. First, if an EM counterpart is found, it typically provides a precise sky position. Second, the mass parameters, which are inferred from the phasing of the GW waveform, do not significantly couple to the distance and inclination, which are primarily measured from the amplitude of the signal. Since the arrival time, arrival phase (or, rather, the arrival time and phase difference between detector pairs), and the signal-to-noise ratio are measured, the only unknown parameter left is the orientation of the binary in the plane of sky, $\psi$ ("polarization") [68]. We modify the Bayesian estimator of Ref. [69] to use the events' signal-to-noise ratios, the relative arrival time differences, and the relative phase differences to reconstruct posteriors for luminosity distance and viewing angle while numerically marginalizing over the polarization (see the Appendix for more details).

We verify that the standard deviations we obtain for the viewing angle using the approximate code are very similar to the estimates obtained with LALINFERENCE for the sources described in the previous section. For example, in Fig. 4, we show the uncertainties for source $D$ when the sky position is known or when both the luminosity distance and sky position are known.

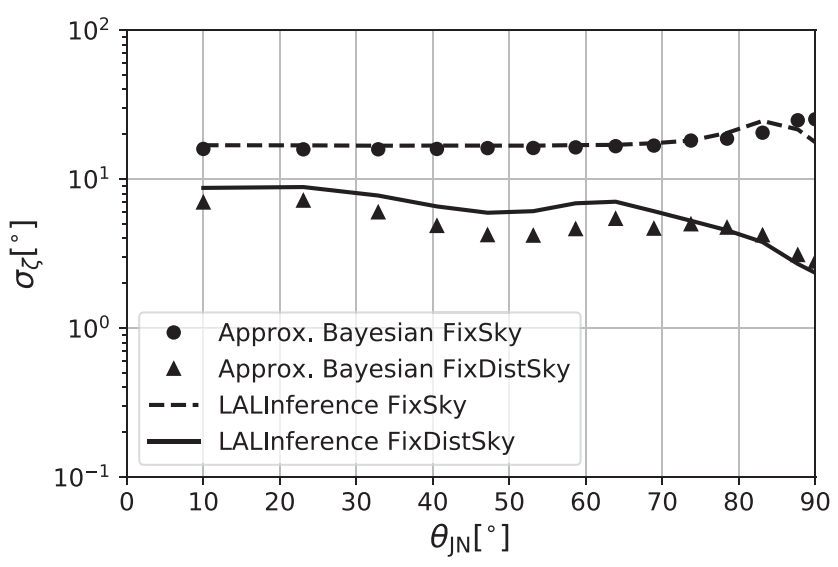

FIG. 4. Standard deviation for the viewing angle of source $D$ against the true value of the inclination angle as measured with the full LALINFERENCE code (lines) and with the approximate Bayesian estimator (symbols). Triangles correspond to the case when both the distance and sky position are known, while circles assume that only the sky position is known. 


\section{Population analysis}

Having shown that the approximate Bayesian estimator gives results which are consistent with LALINFERENCE, we proceed and use the former for large populations of BNSs. We consider three observing scenarios [70]: (i) a network with the two LIGOs and Virgo (HLV) at the expected LIGOVirgo third observing run (O3) sensitivity (approximately 2019+); (ii) HLV at the design sensitivity (approximately 2021+); and (iii) to check how the results evolve as the network of ground-based detectors grows, we also consider a five-detector network made of the two LIGOs, Virgo, LIGO India [71], and Kagra [72,73] (HLVJI), all at their design sensitivity (approximately 2024+). For each scenario, we simulate 1.4-1.4 $\mathrm{M}_{\odot}$ BNSs with random orientation, distributed uniformly in comoving volume. Throughout this work, a standard $\Lambda \mathrm{CDM}$ Planck cosmology is assumed: $\Omega_{M_{0}}=0.3065, \Omega_{\Lambda_{0}}=0.6935$, and $h_{0}=0.679$ [74]. A BNS is considered detected if the measured network signal-tonoise ratio [75] [76] is greater than 12. Following the approach of Ref. [69], we add Gaussian noise to the measured SNR ratio, relative arrival times, and relative phases.

We estimate the distance and inclination for 1000 detections, for each network.

(i) Measurement of the viewing angle.-We first discuss the measurability of the viewing angle using auxiliary EM information (sky position and redshift).

For the simulations in which we assume redshift information exists, we convert the redshift to luminosity distance using the Planck cosmological parameters and marginalize the posterior over the luminosity distance with a Dirac $\delta$ centered at the true distance. We note that, in practice, this conversion might suffer from two sources of uncertainty: The redshift may not be a true measure of the luminosity distance due to the peculiar motion of the source, and the value of the Hubble constant is not precisely known. To account for the former, we introduce a $250 \mathrm{~km} / \mathrm{s}$ Gaussian uncertainty around the true value of the source redshift, which represents a typical uncertainty after the group velocity is corrected [77,78]. We also use a top-hat prior on the Hubble constant, from 65 to $75 \mathrm{~km} / \mathrm{s} / \mathrm{Mpc}$ to cover the range of currently estimated values [74,79]. Other cosmological parameters do not play a significant role for the redshift conversion, given that advanced detectors will detect a BNS only up to a redshift of $z<0.1$.

In Fig. 5, we show the cumulative distribution for the $1 \sigma$ viewing angle uncertainty, for the HLV network at design sensitivity. We see that if the sky positions and redshifts of the BNSs are precisely known, the viewing angle for half of them is constrained to $<6^{\circ}$. This uncertainty increases by approximately $1^{\circ}$ if we include uncertainties in the peculiar velocity and in the cosmology.

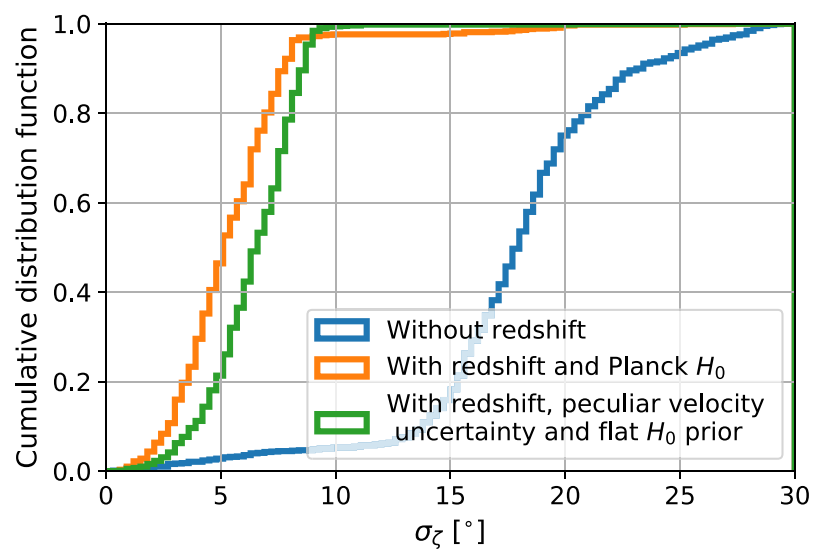

FIG. 5. Cumulative distribution function of $1 \sigma$ viewing angle uncertainty for BNS detected by Advanced LIGO-Virgo at design sensitivity. The sky positions for all events in the distributions are assumed to be precisely known.

If, instead, only sky positions are identified, without any information about the luminosity distance, only $<6 \%$ of BNSs have an inclination uncertainty below $10^{\circ}$. These numbers are comparable for $\mathrm{O} 3$ and approximately $10 \%$ better when the detector network extends to HLVJI.

(ii) Measurement of the luminosity distance.-Next, we wish to explore the situation where the EM sector provides a measurement or bound on the viewing angle and show how that can be used to measure more precisely the luminosity distance of the binary using GW data. We consider various possibilities for the quality of the EM-based $\zeta$ measurement: the ideal scenario, in which the viewing angle is precisely measured; an uncertain measurement; and an upper bound. In the first case, we use a Dirac $\delta$ centered at the true viewing angle as the prior for the inclination angle. If an uncertain measurement is available, we treat the $\zeta$ prior in the GW analysis as a normal distribution centered at the true value and consider different widths of the distribution. Last, we consider the case that the EM sector provides only an upper bound on the viewing angle $\zeta \leq \theta_{\text {up }}$. This scenario can describe a situation in which a clear jet break is observed in the GRB afterglow and the GRB jet is aligned with the binary axis, or any other measurement that might provide an upper limit on the viewing angle.

In Fig. 6, we show the cumulative distribution of the $1 \sigma$ fractional luminosity distance uncertainty for simulated events detected by HLV at the design sensitivity. The blue curve shows the results for the worst-case scenario, when no viewing angle information is available. The green curves are obtained by assuming an uncertain Gaussian measurement from the EM sector, with the standard deviation given in the legend. Finally, the orange line is the optimal 


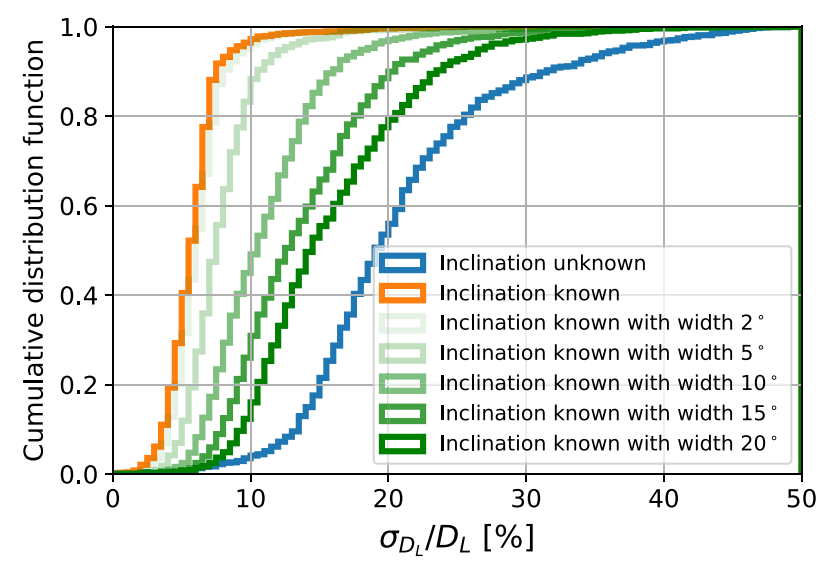

FIG. 6. Cumulative distribution function of the fractional luminosity distance uncertainty, which is calculated as the $1 \sigma$ uncertainty divided by the true value, for BNSs detected by Advanced LIGO-Virgo at design sensitivity.

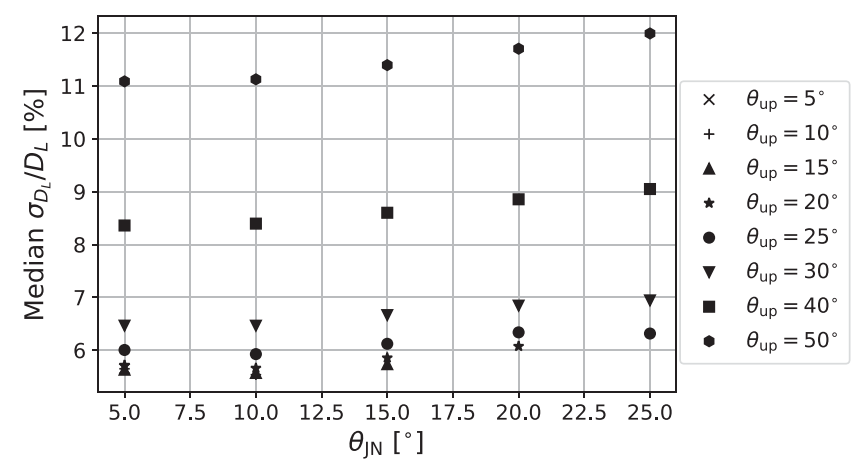

FIG. 7. Median value of the fractional luminosity distance uncertainty assuming the viewing angle is bounded by the EM observations of BNSs detected by Advanced LIGO-Virgo at design sensitivity. Each point represents the median uncertainty of 1000 BNSs with inclination angle $\theta_{\mathrm{JN}}$ and an EM upper bound of $\theta_{\text {up }}$.

situation in which the viewing angle is perfectly known. In this case, the bound on the luminosity distance would be improved by a factor of approximately 3 compared to the worst-case scenario.

In Fig. 7, we show results for the case when an upper bound $\theta_{\text {up }}$ is available for the viewing angle. We consider various combinations of $\left(\theta_{\mathrm{JN}}, \theta_{\mathrm{up}}\right)$, and for each pair we simulate 1000 detections. We show the median fractional luminosity distance uncertainty for the 1000 BNSs having the value of $\theta_{\mathrm{JN}}$ and $\theta_{\mathrm{up}}$ given in the $x$ axis and in the legend. We find that, as long as $\theta_{\text {up }}<30^{\circ}$, the fractional luminosity distance uncertainty is $5 \%-7 \%$ (depending on $\theta_{\mathrm{JN}}$ ).

\section{DISCUSSION}

In this paper, we show that $50 \%$ of the BNSs detected by Advanced LIGO + Virgo for which the sky position and redshift are known (from EM observations) will yield a $1 \sigma$ uncertainty on the viewing angle of $7^{\circ}$ or less (see Fig. 5). We emphasize that this result is independent of any EM emission model. It therefore implies that the viewing angle measurements obtained this way can be used to constrain the EM mechanism. The sky location and redshift of a BNS can be solely determined by the EM counterparts or, in some spectacular events, solely by the host [80] - this is because in some well-localized events the host groups can be uniquely identified without the help of EM counterparts.

We show that BNSs without independent redshift information yield uninteresting inclination constraints unless the system is close to edge on (see Figs. 1 and 6), which is because the distance-inclination degeneracy dominates the uncertainty. Without an independent estimate of the redshift (and hence of the luminosity distance), the degeneracy can be broken only when the binary is close to edge on. Unfortunately, edge-on binaries are harder to detect and localize than face-on binaries; only approximately $3 \%$ of events have a viewing angle $>80^{\circ}$. Even after advanced detectors start observing one BNS a week, only one or two events per year will be close to edge on. And yet, there are several reasons why binaries with large orbital inclinations should be sought out, beside yielding better inclination and luminosity distance measurements (Fig. 1 and Ref. [63]). For example, simulations suggest that there may be interesting EM features along the equatorial plane [18]. EM follow-up observations will have to be properly planned in order to find the counterparts for these rare but valuable sources.

Adding two detectors to the network improves the uncertainty of the viewing angle by only approximately $10 \%$, because the uncertainty on the viewing angle is limited not only by how well one can measure the two polarizations, but also by the very correlation between the luminosity distance and viewing angle, which is significant for sources with small viewing angles, which are the majority. Since HLV can already measure two polarizations, and more detectors will not help break the luminosity distanceviewing angle degeneracy, the uncertainty will not decrease much with larger networks. The only way to improve the measurement is to reduce the luminosity distance-viewing angle degeneracy, which can be done if the system shows amplitude modulation [24,63] or if the distance can be constrained by other means, e.g., an EM counterpart.

The jet break in short GRB afterglows has long been used to study the jet opening angle [36]. The uncertainties in the inferred opening angle are usually a few degrees. It is interesting to verify how well GWs can constrain the BNS's viewing angle for those sources for which a GRB could be detected. To answer this question, we select a subset of the BNSs in Fig. 5, keeping only the sources with $\theta_{\mathrm{JN}}<25^{\circ}$. We find that half of this subsample of sources has a $1 \sigma$ viewing angle uncertainty of $8^{\circ}$ or less, if their sky locations and redshifts are constrained. That level of precision is 
comparable with the jet opening angle uncertainty inferred from the afterglow jet break, allowing for a compelling comparison between the two (under the assumption that the total orbital angular momentum aligns with the jet).

We also show how one can expect a factor of 2-3 improvement in the fractional luminosity distance uncertainty, if the binary inclination angle is independently measured. As the luminosity distance is the main source of uncertainty when measuring the Hubble constant with GWs [81], a better luminosity distance measurement translates into a better $\mathrm{H}_{0}$ measurement. Since the $\mathrm{H}_{0}$ uncertainty scales as $1 / \sqrt{N}[43,82,83]$, where $N$ is the number of detections, a factor of 3 improvement in distance uncertainty implies that a factor of 9 fewer events are required to achieve any given $\mathrm{H}_{0}$ precision. Instead of the 200 BNS detections required, as estimated in Ref. [83], only $\mathrm{O}(10) \mathrm{BNSs}$ would be required to reach a statistical uncertainty of $1 \%$ in $\mathrm{H}_{0}$, if the binary inclination angles were independently constrained. However, we stress that the Hubble constant measurement is subject to systematic errors originating from both $\mathrm{GW}$ and $\mathrm{EM}$ measurements. From GW measurements, the dominating systematics is likely to be the instrumental calibration error in the amplitude, which can potentially lead to a systematic bias in distance estimates [84]. This error is currently around a percent level and is likely to improve [85]. On the other hand, the accuracy of the EM measured binary viewing angle relies on modeling of the EM emission. As more EM observations are made, EM modeling will improve, and those uncertainties will go down. But, at least in the near future, the systematic uncertainty in GW-EM cosmological measurements will likely be dominated by the EM data.

In Fig. 7, we show that the median value of the fractional luminosity distance uncertainty for BNSs can be as small as $5 \%-7 \%$ if the upper bound $\theta_{\text {up }}$ on the viewing angle from EM observation is smaller than $30^{\circ}$. This result is consistent with what is found by Ref. [38]. We further find that, as long as the upper bound is smaller than $30^{\circ}$, the improvement in the luminosity distance measurement does not depend strongly on how tight the bound is. Conversely, the luminosity distance uncertainty is not significantly improved if the upper bound is larger than $30^{\circ}$, which is because, as shown in the single-event analysis section, the viewing angle posteriors are equal to the Schutz distribution for true viewing angle smaller than approximately $70^{\circ}$. Since the Schutz distribution peaks around $30^{\circ}$ (black dashed line in Fig. 2), an EM-based upper limit helps only if it bounds the viewing angle to be smaller than approximately $30^{\circ}$. Fortunately, whenever an upper bound on the viewing angle is provided by a jet-break observation, the inferred jet opening angle of the short GRB almost never exceeds $30^{\circ}[33,36,86-92]$. We thus expect that observations of short GRB jet breaks will significantly improve the luminosity distance measurement for BNSs.

\section{ACKNOWLEDGMENTS}

We acknowledge valuable discussions with K. G. Arun, Juan Calderon Bustillo, Maria Haney, Daniel Holz, Vivien Raymond, Om Sharan Salafia, B. S. Sathyaprakash, and John Veitch. H.-Y.C. was supported by the Black Hole Initiative at Harvard University, through a grant from the John Templeton Foundation. S. V. acknowledges support of the MIT physics department through the Solomon Buchsbaum Research Fund, the National Science Foundation, and the LIGO Laboratory. LIGO was constructed by the California Institute of Technology and Massachusetts Institute of Technology with funding from the National Science Foundation and operates under Cooperative Agreement No. PHY-1764464. R. N. was supported in part by NSF Grant No. AST1816420. The authors acknowledge the LIGO Data Grid clusters. We are grateful for computational resources provided by Cardiff University and funded by an STFC grant supporting United Kingdom Involvement in the Operation of Advanced LIGO. We acknowledge useful comments from the anonymous referees. LIGO Document No. P1800196.

\section{APPENDIX}

\section{LALINFERENCE inclination angle posteriors}

In Figs. 2, 8-10, we show the posteriors on the viewing angle obtained with LALINFERENCE when no EM information is provided. Thin lines (all overlapping) correspond to small and moderate inclination angles. We use thicker lines for sources with $\theta_{\mathrm{JN}}=74^{\circ}, 78^{\circ}, 83^{\circ}, 88^{\circ}, 90^{\circ}$. For these sources, the viewing angle posterior is usually significantly different from the prior. We stress that source $A$ is quite loud, with a network SNR of 35 , similar to GW170817 [1]. For the weakest event, source $D$, the SNR is so low that the two polarizations cannot be disentangled even partially, and the posteriors are exactly equal to the Schutz distribution, with an uncertainty of $19.5^{\circ}$ for small and moderate inclinations (the standard deviation on the

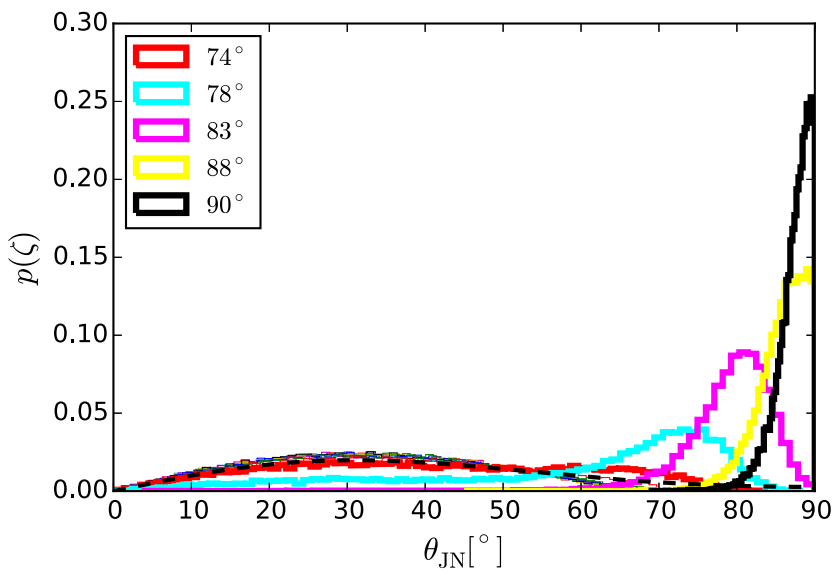

FIG. 8. The same as Fig. 2, but for source $A$. 


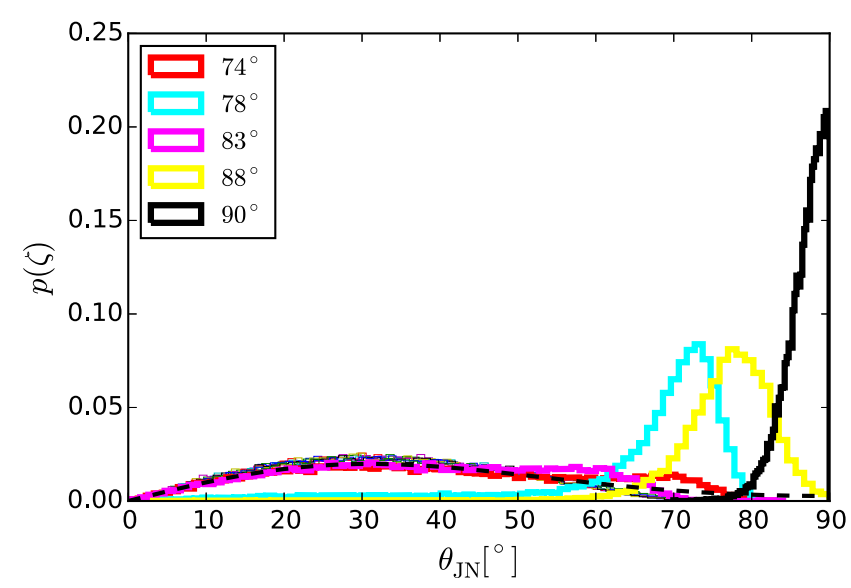

FIG. 9. The same as Fig. 2, but for source $C$.

viewing angle of the Schutz distribution, inferred from the analytic expression in Ref. [44], is $19.4^{\circ}$ ).

\section{Approximate Bayesian estimator}

We use each individual detector's signal-to-noise ratio, $\vec{\rho}=\left(\rho_{a}, \rho_{b}, \rho_{c} \ldots\right)[a, b, c$, etc., denote different detectors $]$, the relative arrival time difference between detector pairs, $\overrightarrow{\Delta t}=\left(\Delta t_{a b}, \Delta t_{a c}, \ldots\right),[93]$ and the relative phase difference between detector pairs, $\overrightarrow{\Delta \eta}=\left(\Delta \eta_{a b}, \Delta \eta_{a c}, \ldots\right)$, to reconstruct the distance $D$ and inclination $\theta_{\mathrm{JN}}$ posterior:

$$
\begin{aligned}
f\left(D, \theta_{\mathrm{JN}} \mid \vec{\rho}, \overrightarrow{\Delta t}, \overrightarrow{\Delta \eta}\right) \propto f\left(D, \theta_{\mathrm{JN}}\right) f\left(\vec{\rho}, \overrightarrow{\Delta t}, \overrightarrow{\Delta \eta} \mid D, \theta_{\mathrm{JN}}\right) \\
=f\left(D, \theta_{\mathrm{JN}}\right) \frac{1}{f\left(D, \theta_{\mathrm{JN}}\right)} \\
\quad \times \int f\left(\vec{\rho}, \overrightarrow{\Delta t}, \overrightarrow{\Delta \eta} \mid D, \theta_{\mathrm{JN}}, \psi\right) f\left(D, \theta_{\mathrm{JN}}, \psi\right) d \psi \\
=\int f\left(\vec{\rho}, \overrightarrow{\Delta t}, \overrightarrow{\Delta \eta} \mid D, \theta_{\mathrm{JN}}, \psi\right) f\left(D, \theta_{\mathrm{JN}}, \psi\right) d \psi .
\end{aligned}
$$

The first line of Eq. (A1) follows the Bayes theorem. $f\left(D, \theta_{\mathrm{JN}}\right)$ is the prior on the distance and the binary

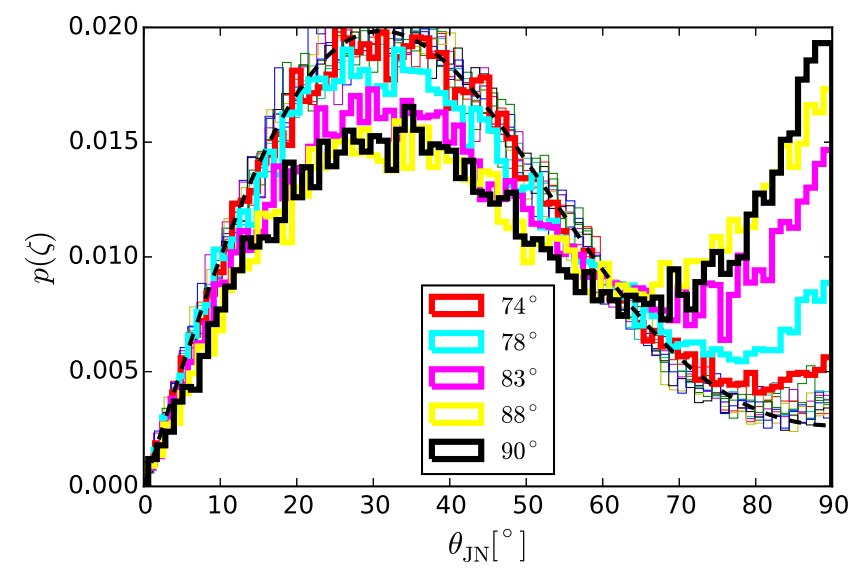

FIG. 10. The same as Fig. 2, but for source $D$. inclination angle, and $f\left(\vec{\rho}, \overrightarrow{\Delta t}, \overrightarrow{\Delta \eta} \mid D, \theta_{\mathrm{JN}}\right)$ is the likelihood. Since the signal-to-noise ratio and the phase difference also depend on the binary orientation angle $\psi$ (we initially do not write the binary orientation out explicitly, because we are not interested in this quantity), we write the likelihood as a marginalization over the orientation angle in the second line of Eq. (A1). We then need a different prior $f\left(D, \theta_{\mathrm{JN}}, \psi\right)$. This prior can be written as

$$
f\left(D, \theta_{\mathrm{JN}}, \psi\right)=D^{2} \sin \theta_{\mathrm{JN}} H\left[D_{h}\left(\theta_{\mathrm{JN}}, \psi\right)-D\right],
$$

where $H\left[D_{h}\left(\theta_{\mathrm{JN}}, \psi\right)-D\right]$ is a Heaviside function that cuts off at the maximum distance $D_{h}\left(\theta_{\mathrm{JN}}, \psi\right)$ a binary with inclination and orientation $\left(\theta_{\mathrm{JN}}, \psi\right)$ can be detected. The new likelihood $f\left(\vec{\rho}, \overrightarrow{\Delta t}, \overrightarrow{\Delta \eta} \mid D, \theta_{\mathrm{JN}}, \psi\right)$ is calculated as $f\left(\vec{\rho}, \overrightarrow{\Delta t}, \overrightarrow{\Delta \eta} \mid D, \theta_{\mathrm{JN}}, \psi\right) \sim \exp \left(-\chi_{\rho}^{2} / 2\right) \exp \left(-\chi_{\zeta}^{2} / 2\right)$, where

$$
\chi_{\rho}^{2}=\frac{\sum_{i}\left[\rho_{\text {measured }, i}-\rho_{i}\left(D, \theta_{\mathrm{JN}}, \psi\right)\right]^{2}}{\sigma_{\rho}^{2}},
$$

where the index $i$ goes through all detectors.

$\sigma_{\rho}^{2}$ and $\chi_{\zeta}^{2}$ are taken from Eq. (10)-(14) of Ref. [69]. We grid the $\left(D, \theta_{\mathrm{JN}}, \psi\right)$ parameter space and evaluate $\chi_{\rho}^{2}, \chi_{\zeta}^{2}$, and $f\left(D, \theta_{\mathrm{JN}}, \psi\right)$ in each grid cell. These terms are thus numerically integrated over $\psi$ to yield the joint distanceinclination posterior, Eq. (A1).

[1] B. P. Abbott, R. Abbott, T. D. Abbott, F. Acernese, K. Ackley, C. Adams, T. Adams, P. Addesso, R. X. Adhikari, V. B. Adya et al., GW170817: Observation of Gravitational Waves from a Binary Neutron Star Inspiral, Phys. Rev. Lett. 119, 161101 (2017).

[2] B. P. Abbott, R. Abbott, T. D. Abbott, F. Acernese, K. Ackley, C. Adams, T. Adams, P. Addesso, R. X. Adhikari, V. B. Adya et al., Multi-messenger Observations of a Binary Neutron Star Merger, Astrophys. J. Lett. 848, L12 (2017).

[3] B. P. Abbott, R. Abbott, T. D. Abbott, F. Acernese, K. Ackley, C. Adams, T. Adams, P. Addesso, R. X. Adhikari, V. B. Adya et al., Gravitational Waves and Gamma-Rays from a Binary Neutron Star Merger: GW170817 and GRB 170817A, Astrophys. J. Lett. 848, L13 (2017).

[4] D. A. Coulter, R. J. Foley, C. D. Kilpatrick, M. R. Drout, A. L. Piro, B. J. Shappee, M. R. Siebert, J. D. Simon, N. Ulloa, D. Kasen, B. F. Madore, A. Murguia-Berthier, Y.-C. Pan, J. X. Prochaska, E. Ramirez-Ruiz, A. Rest, and C. Rojas-Bravo, Swope Supernova Survey 2017a (SSS17a), the Optical Counterpart to a Gravitational Wave Source, Science 358, 1556 (2017).

[5] M. Soares-Santos et al., Dark Energy Survey, and Dark Energy Camera GW-EM Collaboration, The Electromagnetic Counterpart of the Binary Neutron Star Merger LIGO/ Virgo GW170817. I. Discovery of the Optical Counterpart Using the Dark Energy Camera, Astrophys. J. Lett. 848, L16 (2017). 
[6] In the gravitational-wave literature, the inclination angle is usually the angle between the line of sight and the orbital angular momentum. However, following LIGO and Virgo, we report the angle between the line of sight and the total angular momentum. The difference is important only for large and misaligned spins, which are not expected for binary neutron star mergers.

[7] A. Goldstein et al., An Ordinary Short Gamma-Ray Burst with Extraordinary Implications: Fermi-GBM Detection of GRB 170817A, Astrophys. J. Lett. 848, L14 (2017).

[8] K. G. Arun, H. Tagoshi, A. Pai, and C. K. Mishra, Synergy of Short Gamma Ray Burst and Gravitational Wave Observations: Constraining the Inclination Angle of the Binary and Possible Implications for Off-Axis Gamma Ray Bursts, Phys. Rev. D 90, 024060 (2014).

[9] E. Troja et al., The X-Ray Counterpart to the GravitationalWave Event GW170817, Nature (London) 551, 71 (2017).

[10] R. Margutti et al., The Electromagnetic Counterpart of the Binary Neutron Star Merger LIGO/Virgo GW170817. $V$. Rising X-Ray Emission from an Off-Axis Jet, Astrophys. J. Lett. 848, L20 (2017).

[11] G. Hallinan et al., A Radio Counterpart to a Neutron Star Merger, Science 358, 1579 (2017).

[12] K. D. Alexander et al., The Electromagnetic Counterpart of the Binary Neutron Star Merger LIGO/Virgo GW170817. VI. Radio Constraints on a Relativistic Jet and Predictions for Late-Time Emission from the Kilonova Ejecta, Astrophys. J. Lett. 848, L21 (2017).

[13] M. M. Kasliwal et al., Illuminating Gravitational Waves: A Concordant Picture of Photons from a Neutron Star Merger, Science 358, 1559 (2017).

[14] O. Gottlieb, E. Nakar, T. Piran, and K. Hotokezaka, A Cocoon Shock Breakout as the Origin of the $\gamma$-Ray Emission in GW170817, Mon. Not. R. Astron. Soc. 479, 588 (2018).

[15] K. P. Mooley, A. T. Deller, O. Gottlieb, E. Nakar, G. Hallinan, S. Bourke, D. A. Frail, A. Horesh, A. Corsi, and K. Hotokezaka, Superluminal Motion of a Relativistic Jet in the Neutron Star Merger GW170817, Nature (London) 561, 355 (2018).

[16] E. T. H. van Eerten, G. Ryan, R. Ricci, J. M. Burgess, M. Wieringa, L. Piro, S. B. Cenko, and T. Sakamoto, A Year in the Life of GW170817: The Rise and Fall of a Structured Jet from a Binary Neutron Star Merger, arXiv:1808.06617.

[17] G. Ghirlanda et al., Re-solving the Jet/Cocoon Riddle of the First Gravitational Wave with an Electromagnetic Counterpart, Science 363, 968 (2019).

[18] D. Kasen, R. Fernández, and B. D. Metzger, Kilonova Light Curves from the Disc Wind Outflows of Compact Object Mergers, Mon. Not. R. Astron. Soc. 450, 1777 (2015).

[19] P. S. Cowperthwaite et al., The Electromagnetic Counterpart of the Binary Neutron Star Merger LIGO/Virgo GW170817. II. UV, Optical, and Near-infrared Light Curves and Comparison to Kilonova Models, Astrophys. J. Lett. 848, L17 (2017).

[20] V. A. Villar, J. Guillochon, E. Berger, B. D. Metzger, P. S. Cowperthwaite, M. Nicholl, K. D. Alexander, P. K. Blanchard, R. Chornock, T. Eftekhari, W. Fong, R. Margutti, and P. K. G. Williams, The Combined Ultraviolet, Optical, and Near-Infrared Light Curves of the Kilonova Associated with the Binary Neutron Star Merger GW170817: Unified
Data Set, Analytic Models, and Physical Implications, Astrophys. J. Lett. 851, L21 (2017).

[21] N. R. Tanvir et al., The Emergence of a Lanthanide-rich Kilonova Following the Merger of Two Neutron Stars, Astrophys. J. Lett. 848, L27 (2017).

[22] B. P. Abbott, R. Abbott, T. D. Abbott, M. R. Abernathy, F. Acernese, K. Ackley, C. Adams, T. Adams, P. Addesso, R. X. Adhikari et al., Properties of the Binary Black Hole Merger GW150914, Phys. Rev. Lett. 116, 241102 (2016).

[23] T. A. Apostolatos, C. Cutler, G. J. Sussman, and K. S. Thorne, Spin-Induced Orbital Precession and Its Modulation of the Gravitational Waveforms from Merging Binaries, Phys. Rev. D 49, 6274 (1994).

[24] A. Vecchio, LISA Observations of Rapidly Spinning Massive Black Hole Binary Systems, Phys. Rev. D 70, 042001 (2004).

[25] S. Vitale, R. Lynch, J. Veitch, V. Raymond, and R. Sturani, Measuring the Spin of Black Holes in Binary Systems Using Gravitational Waves, Phys. Rev. Lett. 112, 251101 (2014).

[26] S. Vitale, R. Lynch, V. Raymond, R. Sturani, J. Veitch, and P. Graff, Parameter Estimation for Heavy Binary-Black Holes with Networks of Second-Generation GravitationalWave Detectors, Phys. Rev. D 95, 064053 (2017).

[27] P. B. Graff, A. Buonanno, and B. S. Sathyaprakash, Missing Link: Bayesian Detection and Measurement of Intermediate-Mass Black-Hole Binaries, Phys. Rev. D 92, 022002 (2015).

[28] L. London, S. Khan, E. Fauchon-Jones, C. García, M. Hannam, S. Husa, X. Jiménez-Forteza, C. Kalaghatgi, F. Ohme, and F. Pannarale, First Higher-Multipole Model of Gravitational Waves from Spinning and Coalescing BlackHole Binaries, Phys. Rev. Lett. 120, 161102 (2018).

[29] J. Ca. Bustillo, J. A. Clark, P. Laguna, and D. Shoemaker, Tracking Black Hole Kicks from Gravitational Wave Observations, Phys. Rev. Lett. 121, 191102 (2018).

[30] M. Cantiello et al., A Precise Distance to the Host Galaxy of the Binary Neutron Star Merger GW170817 Using Surface Brightness Fluctuations, Astrophys. J. Lett. 854, L31 (2018).

[31] B. P. Abbott, R. Abbott, T. D. Abbott, F. Acernese, K. Ackley, C. Adams, T. Adams, P. Addesso et al. (LIGO Scientific Collaboration, Virgo Collaboration), Properties of the Binary Neutron Star Merger GW170817, Phys. Rev. X 9, 011001 (2019).

[32] D. Finstad, S. De, D. A. Brown, E. Berger, and C. M. Biwer, Measuring the Viewing Angle of GW170817 with Electromagnetic and Gravitational Waves, Astrophys. J. Lett. 860, L2 (2018).

[33] D. N. Burrows, D. Grupe, M. Capalbi, A. Panaitescu, S. K. Patel, C. Kouveliotou, B. Zhang, P. Mészáros, G. Chincarini, N. Gehrels, and R. A. M. Wijers, Jet Breaks in Short Gamma-Ray Bursts. II. The Collimated Afterglow of GRB 051221A, Astrophys. J. 653, 468 (2006).

[34] H.-Y. Chen and D. E. Holz, Gamma-Ray-Burst Beaming and Gravitational-Wave Observations, Phys. Rev. Lett. 111, 181101 (2013).

[35] E. Berger, Short-Duration Gamma-Ray Bursts, Annu. Rev. Astron. Astrophys. 52, 43 (2014).

[36] W. Fong, E. Berger, R. Margutti, and B. A. Zauderer, A Decade of Short-duration Gamma-Ray Burst Broadband 
Afterglows: Energetics, Circumburst Densities, and Jet Opening Angles, Astrophys. J. 815, 102 (2015).

[37] Y. Wu and A. MacFadyen, Constraining the Outflow Structure of the Binary Neutron Star Merger Event GW170817 with Markov-Chain Monte Carlo Analysis, Astrophys. J. 869, 55 (2018).

[38] X. Fan, C. Messenger, and I. S. Heng, Probing Intrinsic Properties of Short Gamma-Ray Bursts with Gravitational Waves, Phys. Rev. Lett. 119, 181102 (2017).

[39] C. Guidorzi et al., Improved Constraints on $\mathrm{H}_{0}$ from a Combined Analysis of Gravitational-wave and Electromagnetic Emission from GW170817, Astrophys. J. Lett. 851, L36 (2017).

[40] K. Hotokezaka, E. Nakar, O. Gottlieb, S. Nissanke, K. Masuda, G. Hallinan, K. P. Mooley, and A. T. Deller, A Hubble Constant Measurement from Superluminal Motion of the Jet in GW170817, arXiv:1806.10596.

[41] J. Veitch et al., Parameter Estimation for Compact Binaries with Ground-Based Gravitational-Wave Observations Using the LALInference Software Library, Phys. Rev. D 91, 042003 (2015).

[42] N. Dalal, D. E. Holz, S. A. Hughes, and B. Jain, Short GRB and Binary Black Hole Standard Sirens as a Probe of Dark Energy, Phys. Rev. D 74, 063006 (2006).

[43] S. Nissanke, D. E. Holz, S. A. Hughes, N. Dalal, and J. L. Sievers, Exploring Short Gamma-ray Bursts as Gravitational-Wave Standard Sirens, Astrophys. J. 725, 496 (2010).

[44] B. F. Schutz, Networks of Gravitational Wave Detectors and Three Figures of Merit, Classical Quantum Gravity 28, 125023 (2011).

[45] J. Veitch and A. Vecchio, Bayesian Coherent Analysis of InSpiral Gravitational Wave Signals with a Detector Network, Phys. Rev. D 81, 062003 (2010).

[46] M. Burgay, N. D’Amico, A. Possenti, R. N. Manchester, A. G. Lyne, B. C. Joshi, M. A. McLaughlin, M. Kramer, J. M. Sarkissian, F. Camilo, V. Kalogera, C. Kim, and D. R. Lorimer, An Increased Estimate of the Merger Rate of Double Neutron Stars from Observations of a Highly Relativistic System, Nature (London) 426, 531 (2003).

[47] K. Stovall et al., PALFA Discovery of a Highly Relativistic Double Neutron Star Binary, Astrophys. J. Lett. 854, L22 (2018).

[48] P. Schmidt, F. Ohme, and M. Hannam, Towards Models of Gravitational Waveforms from Generic Binaries: II. Modelling Precession Effects with a Single Effective Precession Parameter, Phys. Rev. D 91, 024043 (2015).

[49] M. Hannam, P. Schmidt, A. Bohé, L. Haegel, S. Husa, F. Ohme, G. Pratten, and M. Pürrer, Simple Model of Complete Precessing Black-Hole-Binary Gravitational Waveforms, Phys. Rev. Lett. 113, 151101 (2014).

[50] R. Smith, S. E. Field, K. Blackburn, C.-J. Haster, M. Pürrer, V. Raymond, and P. Schmidt, Fast and Accurate Inference on Gravitational Waves from Precessing Compact Binaries, Phys. Rev. D 94, 044031 (2016).

[51] J. Aasi, B. P. Abbott, R. Abbott, T. Abbott, M. R. Abernathy, K. Ackley, C. Adams, T. Adams, P. Addesso et al. (LIGO Scientific Collaboration), Advanced LIGO, Classical Quantum Gravity 32, 074001 (2015).
[52] F. Acernese, M. Agathos, K. Agatsuma, D. Aisa, N. Allemandou, A. Allocca, J. Amarni, P. Astone, G. Balestri, G. Ballardin et al., Advanced Virgo: A Second-Generation Interferometric Gravitational Wave Detector, Classical Quantum Gravity 32, 024001 (2015).

[53] B. P. Abbott, R. Abbott, T. D. Abbott, M. R. Abernathy, F. Acernese, K. Ackley, C. Adams, T. Adams, P. Addesso, R. X. Adhikari et al., Properties of the Binary Black Hole Merger GW150914, Phys. Rev. Lett. 116, 241102 (2016).

[54] H.-Y. Chen, R. Essick, S. Vitale, D. E. Holz, and E. Katsavounidis, Observational Selection Effects with Ground-Based Gravitational Wave Detectors, Astrophys. J. 835, 31 (2017).

[55] M. Maggiore, Gravitational Waves, Volume 1: Theory and Experiments (Oxford University Press, New York, 2007).

[56] B. P. Abbott, R. Abbott, T. D. Abbott, M. R. Abernathy, F. Acernese, K. Ackley, C. Adams, T. Adams, P. Addesso, R. X. Adhikari et al., Binary Black Hole Mergers in the First Advanced LIGO Observing Run, Phys. Rev. X 6, 041015 (2016).

[57] B. P. Abbott, R. Abbott, T. D. Abbott, F. Acernese, K. Ackley, C. Adams, T. Adams, P. Addesso, R. X. Adhikari, V. B. Adya et al., GW170104: Observation of a 50-SolarMass Binary Black Hole Coalescence at Redshift 0.2, Phys. Rev. Lett. 118, 221101 (2017).

[58] B. P. Abbott, R. Abbott, T. D. Abbott, F. Acernese, K. Ackley, C. Adams, T. Adams, P. Addesso, R. X. Adhikari, V. B. Adya et al., GW170608: Observation of a 19 Solarmass Binary Black Hole Coalescence, Astrophys. J. Lett. 851, L35 (2017).

[59] B. P. Abbott, R. Abbott, T. D. Abbott, F. Acernese, K. Ackley, C. Adams, T. Adams, P. Addesso, R. X. Adhikari, V. B. Adya et al., GW170814: A Three-Detector Observation of Gravitational Waves from a Binary Black Hole Coalescence, Phys. Rev. Lett. 119, 141101 (2017).

[60] B. P. Abbott, R. Abbott, T. D. Abbott, S. Abraham, F. Acernese, K. Ackley, C. Adams, R. X. Adhikari, V. B. Adya, C. Affeldt et al., GWTC-1: A Gravitational-Wave Transient Catalog of Compact Binary Mergers Observed by LIGO and Virgo during the First and Second Observing Runs, arXiv:1811.12907.

[61] It is worth mentioning that this selection effect may be resolved as more sensitive detectors come online [62].

[62] S. Vitale, Three Observational Differences for Binary Black Holes Detections with Second- and Third-Generation Gravitational-Wave Detectors, Phys. Rev. D 94, 121501 (R) (2016).

[63] S. Vitale and H.-Y. Chen, Measuring the Hubble Constant with Neutron Star Black Hole Mergers, Phys. Rev. Lett. 121, 021303 (2018).

[64] S. Fairhurst, Source Localization with an Advanced Gravitational Wave Detector Network, Classical Quantum Gravity 28, 105021 (2011).

[65] L. P. Singer, L. R. Price, B. Farr, A. L. Urban, C. Pankow, S. Vitale, J. Veitch, W. M. Farr, C. Hanna, K. Cannon, T. Downes, P. Graff, C.-J. Haster, I. Mandel, T. Sidery, and A. Vecchio, The First Two Years of Electromagnetic Follow-up with Advanced LIGO and Virgo, Astrophys. J. 795, 105 (2014). 
[66] L. P. Singer and L. R. Price, Rapid Bayesian Position Reconstruction for Gravitational-Wave Transients, Phys. Rev. D 93, 024013 (2016).

[67] S. Vitale and M. Zanolin, Application of Asymptotic Expansions for Maximum Likelihood Estimators' Errors to Gravitational Waves from Inspiraling Binary Systems: The Network Case, Phys. Rev. D 84, 104020 (2011).

[68] B. S. Sathyaprakash and B. F. Schutz, Physics, Astrophysics and Cosmology with Gravitational Waves, Living Rev. Relativity 12, 2 (2009).

[69] H.-Yu Chen and D.E. Holz, Facilitating Follow-up of LIGO-Virgo Events Using Rapid Sky Localization, Astrophys. J. 840, 88 (2017).

[70] B. P. Abbott, R. Abbott, T. D. Abbott, M. R. Abernathy, F. Acernese, K. Ackley, C. Adams, T. Adams, P. Addesso, R. X. Adhikari et al., Prospects for Observing and Localizing Gravitational-Wave Transients with Advanced LIGO, Advanced Virgo and KAGRA, Living Rev. Relativity 21, 3 (2018).

[71] B. Iyer et al., LIGO-India, Proposal of the Consortium for Indian Initiative in Gravitational-Wave Observations (IndIGO), LIGO-India technical report, 2011.

[72] K. Somiya, Detector Configuration of KAGRA-The Japanese Cryogenic Gravitational-Wave Detector, Classical Quantum Gravity 29, 124007 (2012).

[73] Y. Aso, Y. Michimura, K. Somiya, M. Ando, O. Miyakawa, T. Sekiguchi, D. Tatsumi, and H. Yamamoto, Interferometer Design of the KAGRA Gravitational Wave Detector, Phys. Rev. D 88, 043007 (2013).

[74] P. A. R. Ade, N. Aghanim, M. Arnaud, M. Ashdown, J. Aumont, C. Baccigalupi, A. J. Banday, R. B. Barreiro, J. G. Bartlett et al. (Planck Collaboration), Planck 2015 Results. XIII. Cosmological Parameters, Astron. Astrophys. 594, A13 (2016).

[75] Root sum square of the individual detector's signal-to-noise ratio.

[76] H.-Y. Chen, D. E. Holz, J. Miller, M. Evans, S. Vitale, and J. Creighton, Distance Measures in Gravitational-Wave Astrophysics and Cosmology, arXiv:1709.08079.

[77] J. Carrick, S. J. Turnbull, G. Lavaux, and M. J. Hudson, Cosmological Parameters from the Comparison of Peculiar Velocities with Predictions from the $2 M++$ Density Field, Mon. Not. R. Astron. Soc. 450, 317 (2015).

[78] D. M. Scolnic et al., The Complete Light-Curve Sample of Spectroscopically Confirmed SNe Ia from Pan-STARRS1 and Cosmological Constraints from the Combined Pantheon Sample, Astrophys. J. 859, 101 (2018).

[79] A. G. Riess, L. M. Macri, S. L. Hoffmann, D. Scolnic, S. Casertano, A. V. Filippenko, B. E. Tucker, M. J. Reid, D. O. Jones, J. M. Silverman, R. Chornock, P. Challis, W. Yuan, P. J. Brown, and R. J. Foley, A 2.4\% Determination of the Local Value of the Hubble Constant, Astrophys. J. 826, 56 (2016).
[80] H.-Y. Chen and D. E. Holz, Finding the One: Identifying the Host Galaxies of Gravitational-Wave Sources, arXiv:1612 .01471 .

[81] B. F. Schutz, Determining the Hubble Constant from Gravitational Wave Observations, Nature (London) 323, 310 (1986).

[82] S. Nissanke, D. E. Holz, N. Dalal, S. A. Hughes, J. L. Sievers, and C. M. Hirata, Determining the Hubble Constant from Gravitational Wave Observations of Merging Compact Binaries, arXiv:1307.2638.

[83] H.-Yu Chen, M. Fishbach, and D. E. Holz, A Two Per Cent Hubble Constant Measurement from Standard Sirens within Five Years, Nature (London) 562, 545 (2018).

[84] S. Vitale, W. Del Pozzo, T. G. F. Li, C. Van Den Broeck, I. Mandel, B. Aylott, and J. Veitch, Effect of Calibration Errors on Bayesian Parameter Estimation for Gravitational Wave Signals from Inspiral Binary Systems in the Advanced Detectors Era, Phys. Rev. D 85, 064034 (2012).

[85] S. Karki et al., The Advanced LIGO Photon Calibrators, Rev. Sci. Instrum. 87, 114503 (2016).

[86] M. Ruffert and H.-T. Janka, Gamma-Ray Bursts from Accreting Black Holes in Neutron Star Mergers, Astron. Astrophys. 344, 573 (1999).

[87] M. A. Aloy, H.-T. Janka, and E. Müller, Relativistic Outflows from Remnants of Compact Object Mergers and Their Viability for Short Gamma-Ray Bursts, Astron. Astrophys. 436, 273 (2005).

[88] S. Rosswog, From Neutron Star Binaries to GammaRay Bursts, Nuovo Cimento Soc. Ital. Fis. C 28, 607 (2005).

[89] D. Grupe, D. N. Burrows, S. K. Patel, C. Kouveliotou, B. Zhang, P. Mészáros, R. A. M. Wijers, and N. Gehrels, Jet Breaks in Short Gamma-Ray Bursts. I. The Uncollimated Afterglow of GRB 050724, Astrophys. J. 653, 462 (2006).

[90] L. Rezzolla, B. Giacomazzo, L. Baiotti, J. Granot, C. Kouveliotou, and M. A. Aloy, The Missing Link: Merging Neutron Stars Naturally Produce Jet-like Structures and Can Power Short Gamma-ray Bursts, Astrophys. J. Lett. 732, L6 (2011).

[91] E. Troja, T. Sakamoto, S. B. Cenko, A. Lien, N. Gehrels, A. J. Castro-Tirado, R. Ricci, J. Capone, V. Toy, A. Kutyrev, N. Kawai, A. Cucchiara, A. Fruchter, J. Gorosabel, S. Jeong, A. Levan, D. Perley, R. Sanchez-Ramirez, N. Tanvir, and S. Veilleux, An Achromatic Break in the Afterglow of the Short GRB 140903A: Evidence for a Narrow Jet, Astrophys. J. 827, 102 (2016).

[92] Z.-P. Jin, X. Li, H. Wang, Y.-Z. Wang, H.-N. He, Q. Yuan, F.-W. Zhang, Y.-C. Zou, Y.-Z. Fan, and D.-M. Wei, Short GRBs: Opening Angles, Local Neutron Star Merger Rate, and Off-Axis Events for GRB/GW Association, Astrophys. J. 857, 128 (2018).

[93] $\Delta t_{b c}$ can be obtained as the difference of $\Delta t_{a b}$ and $\Delta t_{a c}$. 